\title{
Motor control strategies during double leg squat following anterior cruciate ligament rupture and reconstruction: an observational study
}

Paulien E Roos ${ }^{1,2^{*}}$, Kate Button ${ }^{1,2,3}$ and Robert W M van Deursen ${ }^{1,2}$

\begin{abstract}
Background: Anterior cruciate ligament (ACL) injured individuals often show asymmetries between the injured and non-injured leg. A better understanding of the underlying motor control could help to improve rehabilitation. Double leg squat exercises allow for compensation strategies. This study therefore investigated motor control strategies during a double leg squat with the aim to investigate if individuals with $A C L$ rupture (ACLD), $A C L$ reconstruction (ACLR) and healthy control subjects (CONT) used different strategies.

Methods: 20 ACLD and 21 ACLR were compared to 21 CONT subjects. Participants performed eight continuous double leg squats to their maximum depth, while kinematic and kinetic data were collected. Outcome measures were calculated to quantify the behavior of the injured and non-injured legs and the asymmetry between these legs.

Results: Squat depth was significantly reduced in ACLR and ACLD compared to CONT $\left(p<0.05 ; 106 \pm 17^{\circ} ; 105 \pm\right.$ $\left.21^{\circ} ; 113 \pm 21^{\circ}\right)$. Peak knee extensor moments $\left(M_{k n(m x)}\right)$ were significantly reduced in ACLR and ACLD compared to CONT in the injured leg only $(p<0.05 ; 0.045 \pm 0.015 ; 0.046 \pm 0.016 ; 0.059 \pm 0.022$ body weight.height respectively). There was no significant correlation between symmetry of the support moment (SYM Msup) and of the \% support moment by the knee $\left(S Y M \%\right.$ sup $\left.k_{n}\right)$ in CONT $\left(R^{2}=-0.07\right)$. Data distribution average indicated good symmetry. ACLR showed a significant correlation between SYM Msup and SYM\%sup $k n\left(R^{2}=0.561\right)$ when two participants who did not recover as well were excluded. ACLR controlled knee moment magnitude using two strategies; 1 ) transfer of support moment to non-injured leg; 2) transfer of support moment from knee to ankle and/or hip of injured leg. These were combined in different proportions, but with the same effect on the knee moment. ACLD showed no significant correlation between SYM Msup and SYM\%sup $k n\left(R^{2}=0.015\right)$. Data distribution average indicated reduced symmetry. ACLD therefore used an avoidance strategy: reducing squat depth and subsequently the support moment in the injured leg and the knee contribution.
\end{abstract}

Conclusions: ACLD and ACLR individuals used different squatting strategies compared to controls, with ACLR using controlled and ACLD using avoidance behavior regarding knee loading. This has major implications for rehabilitation as these kinetic strategies cannot be observed, but result in the injured leg not being exercised as intended.

Keywords: Anterior cruciate ligament, Biomechanics, Rehabilitation, Knee, Squat

\footnotetext{
* Correspondence: RoosPE@Cardiff.ac.uk

${ }^{1}$ School of Healthcare Sciences, Cardiff University, Heath Park, Cardiff CF14

$4 \mathrm{XN}$, UK

${ }^{2}$ Arthritis Research UK Biomechanics and Bioengineering Centre, Cardiff

University, Cardiff, UK

Full list of author information is available at the end of the article
} 


\section{Background}

Anterior Cruciate Ligament (ACL) injury often results in failure to return to pre-injury activity levels [1-4]. Approximately $42-65 \%$ of ACL injured individuals who had reconstructive surgery [1-3] and 18-50\% of those who were treated conservatively $[2,4]$ do not return to their pre-injury levels. ACL injury also leads to an increased predisposition of early-onset osteoarthritis [5], affecting $50 \%$ of ACL deficient individuals (ACLD) [6] and 33-39 \% of individuals with ACL reconstruction (ACLR) [7,8]. The mechanisms from injury to early onset osteoarthritis are unknown, although altered loading of the knee has been proposed as one important potential mechanism [9-11]. Better insight into altered kinematics and kinetics during functional activities is therefore needed. Numerous studies have highlighted that there are asymmetries in kinematics or kinetics between the injured and non-injured leg in ACL patients [12-19]. Asymmetries persisted even six months to two years post-surgery $[13,16,17,19]$. Such asymmetries could result in altered loading of the knee joint. Although asymmetric behavior has been reported, insight into the motor control strategies behind the asymmetries is limited. A better understanding of the motor control of these asymmetries should help to improve rehabilitation of ACL injured patients.

Double leg squat exercises are used in early rehabilitation of ACL injured individuals to strengthen quadriceps and hamstring muscles and to inform treatment selection [20-22]. Asymmetric behavior will however limit the effectiveness of this exercise which allows for compensation strategies because it involves multiple joints and bilateral leg support. It appears that ACLR patients adopt such asymmetric strategies during this exercise [14], with a reduced peak knee extensor moment and a trend to an increased peak hip extensor moment in the injured compared to the non-injured leg [14]. The loading response has also been found to be asymmetric in ACLR patients and reduced in the injured compared to the non-injured leg [23]. This suggests a compensation mechanism of reduced effort of the knee and increased effort of the hip in the injured leg. The sample size in Salem et al. [14] was however too small to demonstrate a significantly increased effort of the hip and to investigate the motor control strategies underlying these compensations. The motor control strategies behind the asymmetries during a double leg squat in ACL injured individuals therefore remain not well understood. A better understanding of such strategies is essential to be able to address them in rehabilitation. Furthermore, no studies have investigated double leg squatting strategies in ACLD patients.

This study therefore investigated motor control strategies during a double leg squat with the aim to investigate if individuals with ACL rupture (ACLD), ACL reconstruction (ACLR) and healthy control subjects (CONT) used different strategies. It was hypothesized that both ACLR and ACLD would use compensation strategies during the double leg squat with a decreased effort of the knee and an increased effort of the hip in the injured compared to the non-injured leg and that these strategies would differ between ACLR and ACLD.

\section{Methods \\ Participants}

20 ACLD (height: $1.80 \pm 0.08 \mathrm{~m}$, mass: $82.9 \pm 12.5 \mathrm{~kg}$, age: $29 \pm 6$ years, gender: 3 female, 17 male) and 21 ACLR (height: $1.73 \pm 0.07 \mathrm{~m}$, mass: $80.1 \pm 9.5 \mathrm{~kg}$, age: $29 \pm 9$ years, gender: 5 female, 16 male) were compared to 21 CONT subjects (height: $1.75 \pm 0.13 \mathrm{~m}$, mass: $77.6 \pm 19.6 \mathrm{~kg}$, age: $27 \pm 8$ years, gender: 9 female, 12 male). Participant numbers were based on power calculations; using means and standard deviations from Salem et al. [14] we calculated an effect size [24] of 0.84 , when using an alpha of 0.05 and power of 0.8 this resulted in 21 participants in each group.

There was no significant difference in characteristics ( $\mathrm{p}=0.093, \mathrm{p}=0.506$ and $\mathrm{p}=0.540$ for height, mass and age respectively) between the participant groups. There was no significant difference in time since injury between ACLD and ACLR groups (ACLR: $24 \pm 17$ months; ACLD: $19 \pm 52$ months; $\mathrm{p}=0.693$; Table 1). All ACLR participants had a single bundle four strand gracilissemitendinosus tendon graft reconstruction and were at least 6 months post-surgery. All participants provided informed consent to take part in this study and ethical approval was obtained from South East Wales Local Research Ethics Committee. Inclusion criteria were that ACLR and ACLD patients were aged between 18 and 65 years; had an ACL rupture that may or may not be accompanied with a meniscal tear or collateral ligament sprain; had a primary ACL reconstruction (for ACLR group only); had finished their rehabilitation; had no other pathology which affects their movement; had no previous knee surgery and were able to provide informed consent independently. Inclusion criteria for CONT were that subjects were aged between 18 and 65 years; had no knee injury, knee surgery or other pathology which affects their movement and were able to provide informed consent independently.

\section{Patient rated questionnaires and strength measurement} For ACLD and ACLR subjects knee-specific symptoms, function and sports activity was scored as a single measure using the International Knee Documentation Subjective Knee $(I K D C)$ questionnaire [25]. Fear of re-injury was scored for ACLD and ACLR subjects using the Tampa Scale of Kinesiophobia (TSK) [26] with adaptations for 
Table 1 Participant strength and questionnaire data

\begin{tabular}{llllllll}
\hline & $\mathbf{T}_{\text {inj }}$ (mths) & $\mathbf{T}_{\text {surg }}$ (mths) & $\boldsymbol{S}_{\text {KnExt }}$ (BW.h) & $\boldsymbol{S}_{\text {KnFlex }}(\mathbf{B W} . \mathbf{h})$ & CSAS & TSK & IKDC \\
\hline CONT & - & - & $0.105 \pm 0.026$ & $0.061 \pm 0.014$ & $87 \pm 17$ & - \\
ACLR & $24 \pm 17$ & $13 \pm 9$ & $0.096 \pm 0.039$ & $0.057 \pm 0.017$ & $82 \pm 16$ & $32.7 \pm 4.9$ & $83 \pm 10$ \\
ACLD & $19 \pm 52$ & - & $0.083 \pm 0.033^{*}$ & $0.054 \pm 0.016^{*}$ & $72 \pm 19^{*}$ & $41.0 \pm 5.1 \&$ & $61 \pm 12 \&$ \\
\hline
\end{tabular}

Mean time since injury $\left(T_{\text {inj }}\right)$, time since surgery $\left(T_{\text {surg }}\right)$, knee extensor $\left(S_{K n E x t}\right.$ and $\left.S_{K n F l e x}\right)$ and knee flexor strength, Cincinnati Knee Rating System Sports Activity Scale (CSAS), Tampa Scale of Kinesiophobia (TSK) and International Knee Documentation Subjective Knee questionnaire (IKDC) scores with standard deviations. $A *$ indicates a significant difference $(p<0.05)$ from CONT, and a \& significant difference from ACLR $(p<0.05)$.

knee injury specific fear of re-injury as in [27]. Activity level was scored (general for CONT and post-injury for ACLD and ACLR) using item 3 (Sports Activity Scale $(C S A S)$ ) from the Cincinnati Knee Rating System [28]. Isokinetic knee extensor $\left(S_{\text {KnExt }}\right)$ and flexor $\left(S_{\text {KnFlex }}\right)$ strength were measured at $60^{\circ} / \mathrm{s}$ on a Biodex System 4 PRO dynamometer (Biodex Medical Systems Inc, USA). This was measured through the full range of motion and over five repetitions without any rest in between. The subjects were sitting upright with the hip at approximately $90^{\circ}$ flexion and the thigh fixed to the dynamometer chair. Although this was measured on both legs, data are presented for the injured (ACLR and ACLD) and the dominant stance leg (CONT) only.

\section{Data collection and processing}

Participants were asked to perform eight continuous double leg squats to their maximum depth. The feet were placed each on a separate force platform. No further instructions were given on foot placement, upper limb position or squatting technique in order to observe the participants' normal behavior. Prior to this a static anatomical calibration trial was collected. Kinematic data were collected at $250 \mathrm{~Hz}$ using an eight camera VICON MX motion analysis system (Oxford Metrics Group Ltd., UK). Reflective markers were placed using the 'Plug-inGait' full body marker set. Two additional markers were placed on the left and right lateral sides of the iliac crest (LILC and RILC). Ground reaction force data were collected using two Kistler force plates (Kistler Instruments Ltd., Switzerland) at 1,000 Hz. Ground reaction force data were filtered with a fourth order Butterworth filter and a low pass cut off frequency of $12 \mathrm{~Hz}$, and marker data with a fourth order Butterworth filter and a low pass cut off frequency of $20 \mathrm{~Hz}$. In most trials the markers on the left and right anterior superior iliac crests (LASI and RASI) were occluded during the deepest point of the squat; these gaps were filled using a custom written program in Vicon BodyBuilder for Biomechanics (version 1.2, Oxford Metrics Group Ltd., UK) and the data of the LILC and RILC markers. This program reconstructed any of the six marker positions missing in the dynamic trial by exploiting the redundancy and using the exact position of any missing markers relative to a pelvis reference frame defined in the static calibration trial. The knee axes were aligned using the anatomical calibration trial.

\section{Data analysis}

Inverse kinematics and dynamics calculations were performed within Vicon Nexus software (version 1.6.1) and data were further processed and analyzed in Matlab R2010b (The Mathworks Inc., USA). Anthropometric measurements were recorded (height, mass, leg length, knee width and ankle width and used for the inverse dynamic calculations. Output parameters (after Winter [29]) were calculated in Matlab and were as follows, with variables with the subscript ending in I relating to the injured leg and ending in $\mathrm{N}$ to the non-injured leg (in CONT $\mathrm{N}$ and I were randomly taken as the left or right leg): $\alpha_{k n(m x)}$ : peak knee flexion angles; $M_{k n(m x)}$ : peak knee extensor moments; $M_{\text {sup }}$ : support moment (sum of the ankle plantar flexor and knee and hip extensor moments) at $M_{k n(m x)}$; Msup ank the percentage of Msup produced by the ankle; $\operatorname{Msup}_{k n}$ the percentage of Msup produced by the knee; Msup $_{\text {hip }}$ the percentage of $M s u p$ produced by the hip; $S Y M \alpha_{k n(m x)}$ : symmetry of the peak knee flexion angles between the injured and non-injured legs; SYM Msup: symmetry of the support moment between the injured and non-injured legs; SYM\%sup $k$ : symmetry of the \% support moment of the knee between the injured and non-injured legs; SYM\%sup ankle: symmetry of the \% support moment of the ankle between the injured and non-injured legs; SYM\%sup hip: symmetry of the \% support moment of the hip between the injured and non-injured legs. Symmetry was calculated as follows [29]:

$$
\text { Symmetry }=\frac{2 * \text { Injured }}{\text { Injured }+ \text { Non-injured }} * 100
$$

\section{Statistical analysis}

After checking for normal distribution, a one-way ANOVA was used for the normal distributed kinematic and kinetic output variables and a Kruskal-Wallis test for the not normal distributed symmetry measures to investigate differences between ACLR and CONT and between ACLD and CONT. To investigate trends between the symmetry measures simple linear regression analysis 
was used between SYM\%sup ankle and SYM\%sup $k$, and

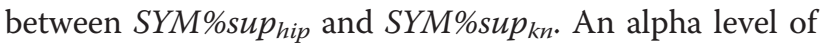
$\mathrm{p}<0.05$ was used to evaluate statistically significant between-groups difference.

\section{Results and discussion}

Participant characteristics

ACLD had significantly lower knee flexor $\left(S_{\text {KnFlex }}\right)$ and extensor strength $\left(S_{K n E x t}\right)$ than CONT $(\mathrm{p}<0.001)$, while strength of ACLR was not significantly different from CONT ( $\mathrm{p}=0.068 ; \mathrm{p}=0.057$ ) when strength was normalized to body weight and height (Table 1). ACLD had a significantly lower activity level than CONT $(\mathrm{p}=0.020$; CSAS; CONT: $87 \pm 17$; ACLR: $82 \pm 16$; ACLD: $72 \pm 19$ ), higher fear of re-injury $(\mathrm{p}=<0.001$; TSK; ACLR: $32.7 \pm 4.9$; ACLD: $41.0 \pm 5.1$ ) and lower knee function $(\mathrm{p}=<0.001$; IKDC; ACLR: $83 \pm 10$; ACLD: $61 \pm 12)$ than ACLR (Table 1). ACLD on average had fear of re-injury while ACLR did not, as a TSK score above 37 has been associated with the presence of fear of re-injury. CONT had a larger proportion of females than ALCD and ACLR. Data were normalized to height and weight to take away the main gender effects. Some gender differences have been reported for limb symmetry, with females being more asymmetric [30]. The smaller proportion of females in the ACLR and ACLD group in our study could therefore have led to an underestimation of asymmetry compared to CONT. Despite this, we still found significant differences regarding asymmetry. It is therefore unlikely that gender differences would have influenced the main conclusions from our study.

\section{Squatting kinematics and kinetics}

A significant difference in performance was shown by a reduced squat depth in both ACLD and ACLR compared to CONT $\left(\alpha_{\mathrm{kn}(\mathrm{mx}) \mathrm{I}}\right.$ : ACLD and ACLR $\mathrm{p}<0.001$ and $\alpha_{\mathrm{kn}(\mathrm{mx}) \mathrm{N}}$ : ACLD $\mathrm{p}=0.025$ ACLR $\mathrm{p}=0.035$; Table 2). In ACLD peak knee extensor moments were significantly reduced compared to CONT in both the injured and the non-injured leg $\left(M_{k n(m x)}: \mathrm{p}=<0.001\right.$ and $M_{k n}$ $\left.(m x) N^{:}: \mathrm{p}=0.041\right)$, while in ACLR this was only significantly reduced in the injured leg $(\mathrm{p}<0.001$; Table 2$)$. Despite the reduced squat depth and peak knee moments in ACLR and ACLD compared to CONT, their total support moment was not significantly reduced compared to CONT (Msup totI $_{\text {and }}$ Msup totN $;_{1} ; \mathrm{p}=0.109$ and $\mathrm{p}=0.152$ respectively; Table 2). This suggests that in ACLR and ACLD the knee contributed to a lesser amount to the support moment compared to CONT. This was confirmed by our results as the percentage contribution of the knee to the total support moment was significantly reduced in the injured leg only in both ACLR and ACLD compared to CONT (Msup knt: ACLD

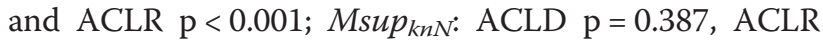
$\mathrm{p}=0.324$; Table 3; Msup $_{k n I}$ : CONT: $44 \pm 12$; ACLR: $36 \pm 10$; ACLD: $36 \pm 9$ ). ACLD showed an increased contribution of the ankle to the support moment compared to CONT in both the injured and non-injured leg, and a decreased contribution of the hip in the non-injured leg (Msup $_{\text {ankb }}$ Msup $_{\text {ankN }}$ and Msup hipN ; Table 3; p <0.001). ACLR showed an increased contribution of the hip joint only in the injured leg (Table 3; $p<0.001$ ). The reduced contribution of the knee was therefore mainly compensated for by an increased contribution of the hip. This was in agreement with findings by Salem et al. [14] in ACLR individuals. The reduction of the peak knee extensor moment in the injured compared to the noninjured leg was similar in ACLR in our study to that by Salem et al. [14] (we found a reduction of $20 \%$ compared to a reduction of $32.6 \%$ by Salem et al.). No studies have investigated this in ACLD individuals.

\section{Limb symmetry}

These results indicate that even though the total support moments did not differ between the groups, there were differences between the injured and non-injured legs (asymmetries). To look further into these asymmetries, symmetry measures between the injured and noninjured leg were investigated. To define whether symmetry was near perfect, the decision rule was used that the rounded symmetry measure had to be 100 . From our own data we have experienced that normal symmetry in healthy people fits within $5 \%$ and our decision rule is meant to be consistent with this [31]. Symmetry of the peak knee flexion angle $\left(S Y M \alpha_{k n(m x)}\right)$ was close to 100 in all groups (CONT: $100 \pm 3$; ACLR: $100 \pm 3$; ACLD: $99 \pm 3$; Table 4) indicating near perfect symmetry between the injured and non-injured leg, as was expected in this closed chain exercise. $S Y M \alpha_{k n}(m x)$ was not significantly different in ACLD and ACLR from CONT (ACLD: $\mathrm{p}=0.057$; ACLR: $\mathrm{p}=1.000$ ). SYM $M_{\text {Msup }}$ was significantly reduced in ACLD $(\mathrm{p}=0.001)$, but not ACLR

Table 2 Kinematics and kinetics

\begin{tabular}{|c|c|c|c|c|c|c|}
\hline & $a_{k n(m x) !}\left(^{\circ}\right)$ & $a_{k n(m x) N}\left({ }^{\circ}\right)$ & $M_{k n(m x) l}(\mathrm{BW} . \mathrm{h})$ & $M_{k n(m x) N}$ (BW.h) & Msup (BW.h) & $\operatorname{Msup}_{N}$ (BW.h) \\
\hline CONT & $113 \pm 21$ & $113 \pm 21$ & $0.059 \pm 0.022$ & $0.060 \pm 0.022$ & $0.143 \pm 0.044$ & $0.144 \pm 0.042$ \\
\hline ACLR & $106 \pm 17^{*}$ & $106 \pm 17^{*}$ & $0.045 \pm 0.015^{*}$ & $0.054 \pm 0.014^{*}$ & $0.134 \pm 0.042$ & $0.140 \pm 0.042$ \\
\hline ACLD & $105 \pm 21^{*}$ & $106 \pm 19^{*}$ & $0.046 \pm 0.016^{*}$ & $0.061 \pm 0.025$ & $0.134 \pm 0.036$ & $0.150 \pm 0.046$ \\
\hline
\end{tabular}

Mean peak knee flexion angles $\left(a_{k n(m \times) l}\right.$ and $\left.a_{k n(m \times) N}\right)$, peak knee extensor moments $\left(M_{k n(m \times) l}\right.$ and $\left.M_{k n(m \times) N}\right)$ and support moments at $M_{k n(m \times)}\left(M s u p_{l}\right.$ and $\left.M s u p_{N}\right)$ in the injured and non-injured leg respectively, with standard deviations for CONT, ACLR and ACLD. A* indicates a significant difference $(p<0.05)$ from CONT. 
Table 3 Contributions of the ankle, knee and hip to the support moment

\begin{tabular}{lllllll}
\hline & Msup $_{\text {ankl }}(\%)$ & Msup $_{\text {ankN }}$ (\%) & Msup $_{\text {knl }}(\%)$ & Msup $_{\text {knN }}$ (\%) & Msup $_{\text {hipl }}$ (\%) & Msup $_{\text {hip }}$ (\%) \\
\hline CONT & $20 \pm 9$ & $19 \pm 9$ & $44 \pm 12$ & $43 \pm 11$ & $38 \pm 9$ & $38 \pm 9$ \\
ACLR & $21 \pm 11$ & $19 \pm 10$ & $36 \pm 10^{*}$ & $41 \pm 11$ & $43 \pm 8^{*}$ & $40 \pm 12$ \\
ACLD & $27 \pm 7^{*}$ & $25 \pm 8^{*}$ & $36 \pm 9^{*}$ & $41 \pm 10$ & $38 \pm 9$ & $34 \pm 9^{*}$ \\
\hline
\end{tabular}

Mean percentage contribution of the ankle, knee and hip joints to the total support moment with standard deviations. With Msup ankl $_{\text {and }}$ Msup ankN the contribution of the ankle, Msup $_{k n l}$ and Msup knN $_{\text {N }}$ the contribution of the knee and Msup hipl and Msup hipN the contribution of the hip in the injured and non-injured leg respectively. $A$ * indicates a significant difference $(p<0.05)$ from CONT.

$(\mathrm{p}=0.329)$, compared to CONT (Table 4). Symmetry of the support moment $\left(S Y M_{M s u p}\right)$ was close to 100 in CONT (99 \pm 10$)$; the support moment was therefore almost identical in both legs. In ACLD SYM Msup was smaller than 100 (95 \pm 12$)$; the support moment was therefore reduced in the injured compared to the noninjured leg. SYM\%sup $p_{k n}$ was significantly reduced in ACLD and ACLR compared to CONT ( $<<0.001$; Table 4). Symmetry of the \% support moment produced by the knee $\left(S Y M \% s u p_{k n}\right)$ was close to 100 in CONT $(99 \pm 9)$; the contribution of the knee to the total support moment was therefore almost identical in the injured and non-injured leg. SYM\%sup $k n$ was lower than 100 for both ACLR and ACLD $(93 \pm 16$ and $92 \pm 13$ respectively); therefore the knee contributed less to the support moment in the injured compared to the non-injured leg. SYM\%sup ankle was significantly reduced in ACLR ( $\mathrm{p}=$ $0.030)$, but not ACLD ( $\mathrm{p}=0.242)$, compared to CONT (Table 4). Symmetry of the \% support moment produced by the ankle $\left(S Y M \%\right.$ sup $\left._{\text {ankle }}\right)$ was 100 in CONT (100 \pm 16); the contribution of the ankle to the total support moment was therefore identical in the injured and noninjured leg. SYM\%sup ankle was higher than 100 for both ACLR and ACLD (105 \pm 18 and $104 \pm 14$ respectively); therefore the ankle contributed more to the support moment in the injured compared to the non-injured leg. SYM\%sup hip was significantly reduced in ACLR ( $\mathrm{p}=$ $0.005)$ and ACLD $(\mathrm{p}<0.001)$, compared to CONT (Table 4). Symmetry of the \% support moment produced by the hip (SYM\%sup hip $)$ was 100 in CONT (100 \pm 11$)$; the contribution of the hip to the total support moment was therefore identical in the injured and non-injured leg. SYM\%sup hip was higher than 100 for both ACLR and ACLD (105 \pm 15 and $106 \pm 12$ respectively); therefore the hip contributed more to the support moment in the injured compared to the non-injured leg. Overall this means that in CONT the total support moment was evenly distributed over the injured and non-injured leg and the ankle, knee and hip contributed to the total support moment in similar amounts in the injured and noninjured legs. In ACLR the total support moment was also similar distributed over the injured and non-injured leg, however the contribution of the knee was reduced and the contribution of the ankle and hip was increased in the injured compared to the non-injured leg. For ACLD the total support moment was reduced in the injured compared to the non-injured leg, also the contribution of the knee was reduced and the contribution of the hip was increased in the injured compared to the non-injured leg. This suggests the use of different motor control strategies in the different groups.

\section{Squatting motor control strategies}

The two main components that were varied in the different motor control strategies were $S Y M_{M \text { sup }}$ and $S Y M \%$ $\sup _{k n}$. We therefore further investigated these motor control strategies in the different participant groups by looking at the relationship between $S Y M_{M \text { sup }}$ and SYM\% $\sup _{k n}$ (Figure 1). There was no significant correlation between these variables in CONT (adjusted $R^{2}=-0.007$, $\mathrm{p}=0.946$; Figure 1A). Data were randomly distributed around a point close to IV (100,100; representing perfect symmetry in both legs, Figure 1). This variability would be expected in normal unconstrained performance. ACLR showed a small but significant correlation between $S Y M_{M \text { sup }}$ and SYM\%sup kn $_{\text {(adjusted }} \mathrm{R}^{2}=0.098, \mathrm{p}$ $<0.001$; Figure 1B). There were however two subjects that were behaving differently from the other ACLR subjects (circled data in Figure 1B). When these data were not included ACLR showed a significant and strong correlation between $S Y M_{M s u p}$ and $S Y M \% s u p_{k n}$ (adjusted $\mathrm{R}^{2}=0.561, \mathrm{p}<0.001$; Figure $\left.1 \mathrm{~B}\right)$ and were distributed

Table 4 Symmetry of kinematics and kinetics

\begin{tabular}{llllll}
\hline & SYMa $_{\boldsymbol{k n}(\boldsymbol{m x})}(\%)$ & SYM $_{\text {Msup }}(\%)$ & SYM\%sup $_{\text {ankle }}(\%)$ & SYM\%sup $_{\boldsymbol{k n}}$ (\%) & SYM\%sup $_{\text {hip }}$ (\%) \\
\hline CONT & $100 \pm 3$ & $99 \pm 10$ & $100 \pm 16$ & $99 \pm 9$ & $100 \pm 11$ \\
ACLR & $100 \pm 3$ & $97 \pm 11$ & $105 \pm 18^{*}$ & $93 \pm 16^{*}$ & $105 \pm 15^{*}$ \\
ACLD & $99 \pm 3$ & $95 \pm 12^{*}$ & $104 \pm 14$ & $92 \pm 12^{*}$ & $106 \pm 12^{*}$ \\
\hline
\end{tabular}

Mean symmetry of the peak knee flexion angles between the injured and non-injured legs $\left(S Y M a_{k n}(m x)\right.$, symmetry of the support moment between the injured and non-injured legs $\left(S Y M_{M s u p}\right)$, symmetry of the \% support moment of the ankle $\left(S Y M \%\right.$ sup ankle), knee $\left(S Y M \%\right.$ sup $\left._{k n}\right)$ and hip $\left(S Y M \%\right.$ sup $\left._{\text {hip }}\right)$ between the injured and non-injured legs with standard deviations for CONT, ACLR and ACLD. A * indicates a significant difference $(p<0.05)$ from CONT. 

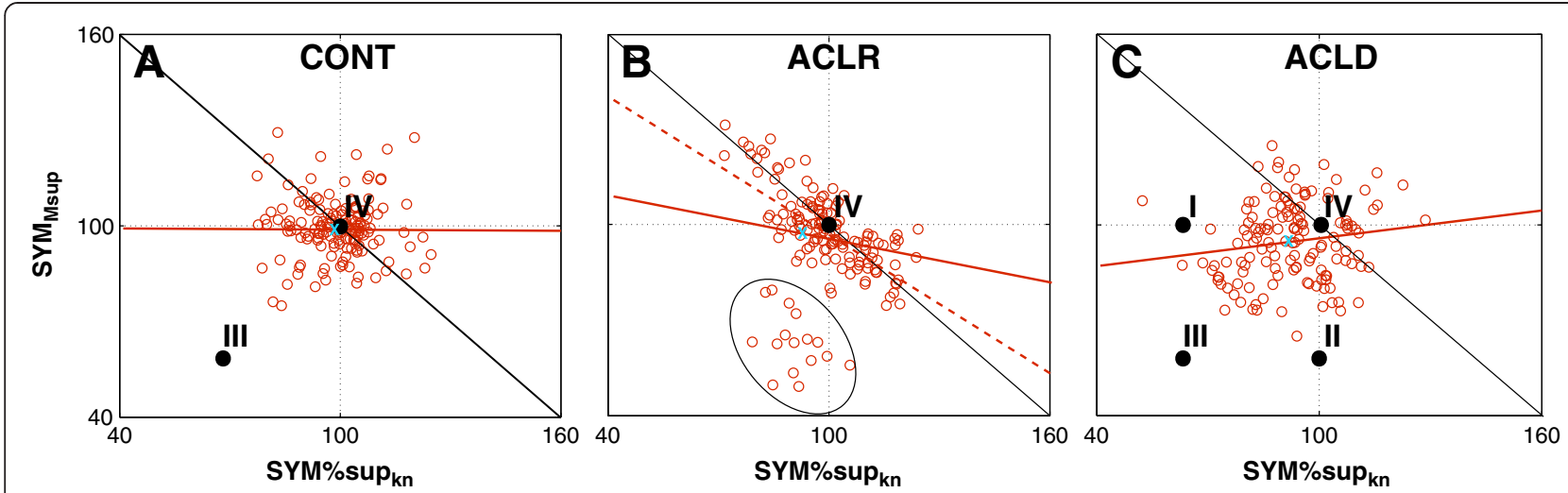

Figure 1 Motor control strategies during double leg squat. SYM $M_{\text {sup }}$ versus SYM\%sup for: A) CONT, B) ACLR and C) ACLD. Combinations below the black solid line have a reduced knee moment in the injured limb. Points I-IV refer to the strategies identified in Figure 2, and the crosses refer to the average group data (Table 4). Adjusted $R^{2}$ : CONT $=-0.007, A C L R$ all data: $0.098, A C L R$ without outliers (dotted line): $0.561, A C L D$ : 0.015. These outliers in ACLR were 2 subjects only.

around a point close to IV $(100,100)$. This was a negative correlation; therefore ACLR participants with the highest $S Y M_{M \text { sup }}$ presented the lowest $S Y M \%$ sup $p_{k n}$ and vice versa. Whilst performing maximally, ACLR seemed constrained by the knee moment on the injured side. ACLR controlled the knee moment magnitude by using two strategies in combination; 1) transfer of support moment to the noninjured leg; 2) transfer of support moment from the knee to the ankle and hip of the injured leg. Different subjects combined these strategies in different proportions. The use of the strategies showed a negative correlation along the diagonal (dashed line in Figure 1B); therefore the increased use of one strategy would reduce the use of the other. The effect on the knee moment was therefore the same among subjects. ACLD showed no significant correlation between $S Y M_{M \text { sup }}$ and $S Y M \%$ sup $p_{k n}$ (adjusted $\mathrm{R}^{2}$ $=0.015, \mathrm{p}=0.080$; Figure $1 \mathrm{C}$ ). The data were distributed around a point below 100 for both $S Y M_{M \text { sup }}$ and $S Y M \%$ $\sup _{k n}$. ACLD therefore used an avoidance strategy where they reduced squat depth and subsequently the support moment in the injured leg and the contribution of the knee to this moment. The lack of correlation could be because some subjects were functioning better than others.

From these results a general performance strategy of reducing depth of squatting and four different motor control strategies could be identified. These are graphically

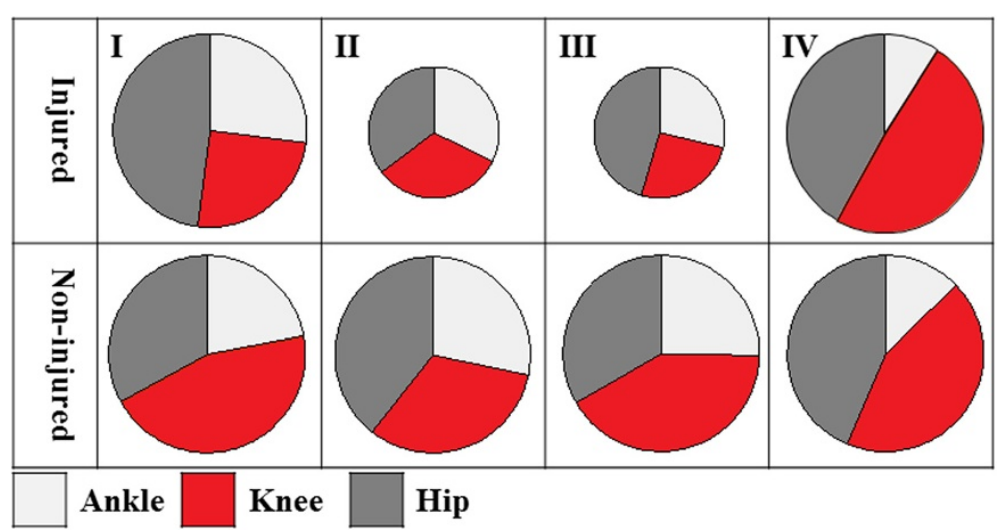

Figure 2 Double leg squat compensation strategies (graphical representation). The slices represent the percentage of support moment produced by the ankle (light grey), knee (red) and hip (dark grey). The sizes of the circles refer to the magnitude of the total support moment; the same size on the injured and non-injured side (I and IV) indicates an even distribution between the legs, while a smaller size circle on the injured side (II and III) indicates a reduced support moment in the injured leg. The strategies I-IV therefore represent the following: I) similar support moment in the injured and non-injured leg but reduced contribution of the knee in the injured leg (some ACLD individuals and ACLR), II) reduced support moment in the injured leg but similar contribution of the knee to the support moment in the injured and non-injured leg (some ACLD individuals and ACLR), III) reduced support moment and reduced contribution of the knee in the injured compared to the noninjured leg (some ACLD individuals only), IV) similar support moment and similar contribution of the knee in the injured and non-injured legs (some ACLD and some ACLR individuals and CONT). 
demonstrated in Figure 2: I) similar support moment in the injured and non-injured leg but reduced contribution of the knee in the injured leg (some ACLD individuals and ACLR), II) reduced support moment in the injured leg but similar contribution of the knee to the support moment in the injured and non-injured leg (some ACLD individuals and ACLR), III) reduced support moment and reduced contribution of the knee in the injured compared to the non-injured leg (some ACLD individuals only), IV) similar support moment and similar contribution of the knee in the injured and non-injured legs (some ACLD and some ACLR individuals and CONT).

\section{Conclusions}

Despite having completed rehabilitation both ACLR and ACLD showed reduced double leg squat performance compared to CONT and used compensation strategies that would reduce loading of their injured knee. Different adaptation of motor control strategies were identified. ACLR demonstrated constrained behavior during a double leg squat to control knee moment magnitude. ACLD used an avoidance strategy with reduced performance, support moment and contribution of the knee to this moment in the injured leg. They both compensated with an increased contribution of the hip and ACLR also with an increased contribution of the ankle. The behavior in ACLD could potentially be explained by their reduced knee extensor and flexor strength and their increased fear of re-injury. The double leg squat is often used in early rehabilitation. This study demonstrated that ACLD and ACLR used different strategies compared to CONT. Attention needs to be paid as these patients may not exercise the injured leg as intended and squat depth on its own may not be adequate as a clinical outcome measure. By observation the exercise can appear normal, while the ACL injured individual may use kinetic compensation strategies. Therefore tools are needed to be able to use biomechanical information in assessment and treatment. These tools could involve manipulation of the support moment or ground reaction forces by means of kinematic adjustments which would be accessible to the clinical setting. The different strategies also highlight that individualized rehabilitation is essential. The long term implications of these findings are unknown. Clearly ACL injured individuals are not recovering movement strategies on early rehabilitation exercises. It could therefore be questioned whether rehabilitation should progress to more demanding exercising before this has been addressed and whether different types of exercises that directly address motor control should be included earlier in rehabilitation.

\section{Abbreviations}

ACL: Anterior Cruciate Ligament; ACLD: Individuals with Anterior Cruciate Ligament deficiency; ACLR: Individuals with Anterior Cruciate Ligament reconstruction; CONT: Healthy control subjects; CSAS: Cincinnati Knee Rating System Sports Activity Scale; IKDC: International Knee Documentation Subjective Knee questionnaire; LASI: Left anterior superior iliac crests; LILC: Left lateral sides of the iliac crest; $M_{k n(m x)}$ : Peak knee extensor moments; $M_{\text {sup }}$ : Support moment at $M_{k n(m x) ;}$ Msup ank: The percentage of Msup produced by the ankle; Msup kn : The percentage of Msup produced by the knee; Msup hip: The percentage of Msup produced by the hip; RASI: Right anterior superior iliac crests; RILC: Right lateral sides of the iliac crest;

$S_{\text {KnExt: }}$ Isokinetic knee extensor strength; $S_{\text {KnFlex: }}$ Isokinetic knee flexor strength; SYM\%sup ankle: Symmetry of the \% support moment of the ankle between the

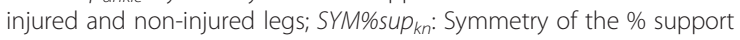
moment of the knee between the injured and non-injured legs; SYM\%

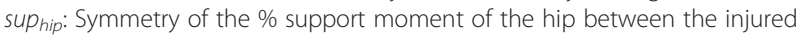
and non-injured legs; SYM Msup: Symmetry of the support moment between the injured and non-injured legs; SYMa ${ }_{k n(m x)}$ : Symmetry of the peak knee flexion angles between the injured and non-injured legs; TSK: Tampa Scale of Kinesiophobia; $a_{k n(m x)}$ : Peak knee flexion angles.

\section{Competing interests}

The authors declare that they have no competing interests.

\section{Authors' contributions}

All authors have made substantial contributions to the conception and design of this research and this manuscript and have provided substantial intellectual input. PR and KB have been involved in data acquisition and processing. KB has provided substantial support with subject recruitment. PR has performed all data analysis and prepared figures and tables for this paper. PR has drafted the manuscript. All authors have been involved in revising the manuscript critically and have given final approval of the version to be published.

\section{Acknowledgements}

Dr. Roos is an Academic Fellow funded by Arthritis Research UK (Grant No: 18461). Dr. Button is funded by National Institute of Social care and Health research, Wales. We further like to acknowledge Dr. Valerie Sparkes for her support in the initial design and supervision of this study, Mr. Rimmer for his support with data collection and subject recruitment and Miss Pooja Swar for her help with data processing. NISCHR CRC for their support with subject recruitment and data collection. These data were first presented at the International Society of Biomechanics 2013 conference.

\section{Author details}

${ }^{1}$ School of Healthcare Sciences, Cardiff University, Heath Park, Cardiff CF14 $4 X N$, UK. ${ }^{2}$ Arthritis Research UK Biomechanics and Bioengineering Centre, Cardiff University, Cardiff, UK. ${ }^{3}$ Cardiff and Vale University Health Board, Cardiff, UK.

Received: 4 June 2013 Accepted: 20 February 2014

Published: 28 February 2014

\section{References}

1. Ardern $\mathrm{CL}$, Webster $\mathrm{KE}$, Taylor NF, Feller JA: Return to sport following anterior cruciate ligament reconstruction surgery: a systematic review and meta-analysis of the state of play. Br J Sports Med 2011, 45:596-606.

2. Myklebust G, Holm I, Maehlum S, Engebretsen L, Bahr R: Clinical, functional, and radiologic outcome in team handball players 6 to 11 years after anterior cruciate ligament injury: a follow-up study. Am J Sports Med 2003, 31:981-989.

3. Gobbi A, Francisco R: Factors affecting return to sports after anterior cruciate ligament reconstruction with patellar tendon and hamstring graft: a prospective clinical investigation. Knee Surg Sports Traumatol Arthrosc 2006, 14:1021-1028.

4. Strehl A, Eggli S: The value of conservative treatment in ruptures of the anterior cruciate ligament (ACL). J Trauma 2007, 62:1159-1162.

5. Blagojevic M, Jinks C, Jeffery A, Jordan KP: Risk factors for onset of osteoarthritis of the knee in older adults: a systematic review and meta-analysis. Osteoarthritis Cartilage 2010, 18:24-33.

6. Lohmander LS, Englund PM, Dahl LL, Roos EM: The long-term consequence of anterior cruciate ligament and meniscus injuries: osteoarthritis. Am J Sports Med 2007, 35:1756-1769. 
7. Hoffelner T, Resch H, Moroder P, Atzwanger J, Wiplinger M, Hitzl W, Tauber $\mathrm{M}$ : No increased occurrence of osteoarthritis after anterior cruciate ligament reconstruction after isolated anterior cruciate ligament injury in athletes. Arthroscopy 2012, 28:517-525.

8. Li RT, Lorenz S, Xu Y, Harner CD, Fu FH, Irrgang JJ: Predictors of Radiographic Knee Osteoarthritis After Anterior Cruciate Ligament Reconstruction. Am J Sports Medicine 2011, 39:2595-2603.

9. Andriacchi TP, Koo S, Scanlan SF: Gait Mechanics Influence Healthy Cartilage Morphology and Osteoarthritis of the Knee. J Bone Joint Surg Am 2009, 91:95-101.

10. Baliunas AJ, Hurwitz DE, Ryals AB, Karrar A, Case JP, Block JA, Andriacchi TP: Increased knee joint loads during walking are present in subjects with knee osteoarthritis. Osteoarthritis Cartilage 2002, 10:573-579.

11. Chaudhari AMW, Briant PL, Bevill SL, Koo S, Andriacchi TP: Knee kinematics, cartilage morphology, and osteoarthritis after ACL injury. Med Sci Sports Exerc 2008, 40:215-222.

12. Gardinier ES, Manal K, Buchanan TS, Snyder-Mackler L: Gait and Neuromuscular Asymmetries after Acute Anterior Cruciate Ligament Rupture. Med Sci Sports Exerc 2012, 44:1490-1496.

13. Castanharo R, da Luz BS, Bitar AC, D'Elia CO, Castropil W, Duarte M: Males still have limb asymmetries in multijoint movement tasks more than 2 years following anterior cruciate ligament reconstruction. J Orthop Sci 2011, 16:531-535.

14. Salem GJ, Salinas R, Harding FV: Bilateral kinematic and kinetic analysis of the squat exercise after anterior cruciate ligament reconstruction. Arch Phys Med Rehabil 2003, 84:1211-1216.

15. Shelbourne KD, Klotz C: What I have learned about the ACL: utilizing a progressive rehabilitation scheme to achieve total knee symmetry after anterior cruciate ligament reconstruction. J Orthop Sci 2006, 11:318-325.

16. Roewer BD, Di Stasi SL, Snyder-Mackler L: Quadriceps strength and weight acceptance strategies continue to improve two years after anterior cruciate ligament reconstruction. J Biomech 2011, 44:1948-1953.

17. Dai B, Butler RJ, Garrett WE, Queen RM: Anterior cruciate ligament reconstruction in adolescent patients. Am J Sports Medicine 2012, 40:2756-2763.

18. Di Stasi SL, Hartigan EH, Snyder-Mackler L: Unilateral stance strategies of athletes with ACL deficiency. Journal of applied biomechanics 2012, 26:374-386

19. Xergia SA, Pappas E, Zampeli F, Georgiou S, Georgoulis A: Asymmetries in functional hop tests, lower extremity kinematics, and isokinetic strength persist 6 to 9 months following anterior cruciate ligament reconstruction. J Orthop Sports Phys Ther 2013, 43(3):154-162

20. Palmitier RA, An KN, Scott SG, Chao EY: Kinetic chain exercise in knee rehabilitation. Sports Med 1991, 11:402-413.

21. Escamilla RF, Macleod TD, Wilk KE, Paulos L, Andrews JR: Anterior cruciate ligament strain and tensile forces for weight-bearing and non-weightbearing exercises: a guide to exercise selection. J Orthop Sports Phys Ther 2012, 42:208-220.

22. Wilk KE, Macrina LC, Cain EL, Dugas JR, Andrews JR: Recent advances in the rehabilitation of anterior cruciate ligament injuries. J Orthop Sports Phys Ther 2012, 42:153-171.

23. Neitzel JA, Kernozek TW, Davies GJ: Loading response following anterior cruciate ligament reconstruction during the parallel squat exercise. Clinical Biomechanics 2002, 17:551-554.

24. Cohen J: Statistical power analysis for the behavioral sciences. Mahwah, NJ, USA: Lawrence Erlbaum Associates; 1988.

25. Irrgang JJ, Anderson AF, Boland AL, Harner CD, Kurosaka M, Neyret P, Richmond JC, Shelborne KD: Development and validation of the international knee documentation committee subjective knee form. Am J Sports Med 2001, 29:600-613.

26. Vlaeyen JW, Kolesnijders AM, Boeren RG, Vaneek H: Fear of movement (re) injury in chronic low-back-pain and its relation to behavioral performance. Pain 1995, 62:363-372.

27. Kvist J, Ek A, Sporrstedt K, Good L: Fear of re-injury: a hindrance for returning to sports after anterior cruciate ligament reconstruction. Knee Surg Sports Traumatol Arthrosc 2005, 13:393-397.

28. Noyes FR, Barber SD, Mooar LA: A rationale for assessing sports activity levels and limitations in knee disorders. Clin Orthop Relat R 1989, 246:238-249.

29. Winter DA: Biomechanics and Motor Control of Human Movement. Hoboken, New Jersey : John Wiley \& Sons, Inc.; 1990.
30. Pappas E, Carpes FP: Lower extremity kinematic asymmetry in male and female athletes performing jump-landing tasks. Journal of Science and Medicine in Sport 2012, 15:87-92.

31. Button $K$, van Deursen $R$, Price $P$ : Measurement of functional recovery in individuals with acute anterior cruciate ligament rupture. $\mathrm{Br} J \mathrm{Sports}$ Med 2005, 39(11):866-871.

doi:10.1186/1743-0003-11-19

Cite this article as: Roos et al:: Motor control strategies during double leg squat following anterior cruciate ligament rupture and reconstruction: an observational study. Journal of NeuroEngineering and Rehabilitation 2014 11:19.

\section{Submit your next manuscript to BioMed Central and take full advantage of:}

- Convenient online submission

- Thorough peer review

- No space constraints or color figure charges

- Immediate publication on acceptance

- Inclusion in PubMed, CAS, Scopus and Google Scholar

- Research which is freely available for redistribution

Submit your manuscript at www.biomedcentral.com/submit
C) Biomed Central 